\title{
Analysis of Type-II Progressively Hybrid Censored Data
}

\author{
DEBASIS KUndu ${ }^{\dagger} \&$ AVIJIT JOARDER ${ }^{\ddagger}$
}

\begin{abstract}
The mixture of Type-I and Type-II censoring schemes, called the hybrid censoring scheme is quite common in life-testing or reliability experiments. Recently Type-II progressive censoring scheme becomes quite popular for analyzing highly reliable data. One drawback of the Type-II progressive censoring scheme is that the length of the experiment can be quite large. In this paper we introduce a Type-II progressively hybrid censoring scheme, where the experiment terminates at a pre-specified time. For this censoring scheme, we analyze the data under the assumptions that the lifetimes of the different items are independent and exponentially distributed random variables with parameter $\lambda$. We obtain the maximum likelihood estimator of the unknown parameter in an exact form. Asymptotic confidence intervals based on $\hat{\lambda}$, $\ln \hat{\lambda}$, confidence interval based on likelihood ratio test and two bootstrap confidence intervals are also proposed. Bayes estimate and credible interval of the unknown parameter are obtained under the assumption of gamma prior of the unknown parameter. Different methods have been compared using Monte Carlo simulations. One real data set has been analyzed for illustrative purposes.
\end{abstract}

Keywords: Maximum likelihood estimator; Type-I and Type-II censoring; Fisher Information matrix; Asymptotic distribution; Bayesian inference; Gamma distribution; Type-II progressive censoring scheme.

$\dagger$ Corresponding Author: Department of Mathematics, Indian Institute of Technology Kanpur, Kanpur, Pin 208016, INDIA. Phone: 91-512-2597141, Fax: 91-512-2597500, e-mail: kundu@iitk.ac.in

$\ddagger$ Reserve Bank of India, Mumbai, India, e-mail: ajoarder@rbi.org.in 


\section{INTRODUCTION}

The two most common censoring schemes are termed as Type-I and Type-II censoring schemes. Briefly, they can be described as follows. Consider $n$ items under observations in a particular experiment. In the conventional Type-I censoring scheme, the experiment continues up to a pre-specified time $T$. On the other hand, the conventional Type-II censoring scheme requires the experiment to continue until a pre-specified number of failures $m \leq n$ occur. The mixture of Type-I and Type-II censoring schemes is known as the hybrid censoring scheme. The hybrid censoring scheme was first introduced by Epstein [9, 10]. But recently it becomes quite popular in the reliability and life-testing experiments. See for example the work of Chen and Bhattacharya [2], Childs et al. [3], Draper and Guttman [7], Fairbanks, Madasan and Dykstra [11], Gupta and Kundu [12] and Jeong, Park and Yum $[14]$

One of the drawbacks of the conventional Type-I, Type-II or hybrid censoring schemes is that they do not allow for removal of units at points other than the terminal point of the experiment. One censoring scheme known as Type-II progressive censoring scheme, which has this advantage, becomes very popular for the last few years. It can be described as follows: Consider $n$ units in a study and suppose $m<n$ is fixed before the experiment. Moreover, $m$ other integers, $R_{1}, \ldots, R_{m}$ are also fixed before so that $R_{1}+\ldots+R_{m}+m=n$. At the time of the first failure, say $X_{1: m: n}, R_{1}$ of the remaining units are randomly removed.

Similarly, at the time of the second failure, say $X_{2: m: n}, R_{2}$ of the remaining units are randomly removed and so on. Finally, at the time of the $m-t h$ failure, say $X_{m: m: n}$, the rest of the $R_{m}$ units are removed. For further details on Type-II progressive censoring and for its different advantages, the readers may refer to the recent excellent monograph of Balakrishnan and Aggarwala [1]. 
In this paper, we introduce a Type-II progressively hybrid censoring scheme. As the name suggests, it is a mixture of Type-II progressive and hybrid censoring schemes. The detail description and its advantages will be described in the next section. In this new censoring scheme, we analyze the data under the assumptions that the lifetimes of the different items are independent identically distributed (i.i.d.) exponential random variables. It is observed that the maximum likelihood estimator (MLE) always exists and we obtain the explicit form of the MLE of the unknown parameter. We also obtain an estimate of the asymptotic confidence interval and propose two bootstrap confidence intervals. Bayes estimate and credible interval are also obtained under the assumption of the gamma prior on the unknown parameter. Different methods are compared using Monte Carlo simulations and for illustrative purposes we analyze one real data set.

The rest of the paper is organized as follows. We formulate the problem in Section 2 and provide the MLE of the unknown parameter. Different confidence intervals are presented in Section 3. Bayesian analysis is provided in Section 4. Numerical results are presented in Section 5. One real data set has been analyzed in Section 6 and finally we conclude the paper in Section 7.

\section{Model Description, Notations and MLE}

\subsection{Model Description And Notations}

Suppose $n$ identical items are put on a test and the lifetime distributions of the $n$ items are denoted by $X_{1}, \ldots, X_{n}$. The integer $m<n$ is fixed at the beginning of the experiment, and $R_{1}, \ldots, R_{m}$ are $m$ pre-fixed integers satisfying $R_{1}+\ldots+R_{m}+m=n$. The time point $T$ is also fixed before hand. At the time of first failure $X_{1: m: n}, R_{1}$ of the remaining units are

randomly removed. Similarly at the time of the second failure $X_{2: m: n}, R_{2}$ of the remaining 
units are removed and so on. If the $m-t h$ failure $X_{m: m: n}$ occurs before the time point $T$, the experiment stops at the time point $X_{m: m: n}$. On the other hand suppose the $m-t h$ failure does not occur before time point $T$ and only $J$ failures occur before the time point $T$, where $0 \leq J<m$, then at the time point $T$ all the remaining $R_{J}^{*}$ units are removed and the experiment terminates at the time point $T$. Note that $R_{J}^{*}=n-\left(R_{1}+\ldots+R_{J}\right)-J$. We denote the two cases as Case I and Case II respectively and call this censoring scheme as the Type-II progressively hybrid censoring scheme. Schematically, it can be represented as in Figure 1.

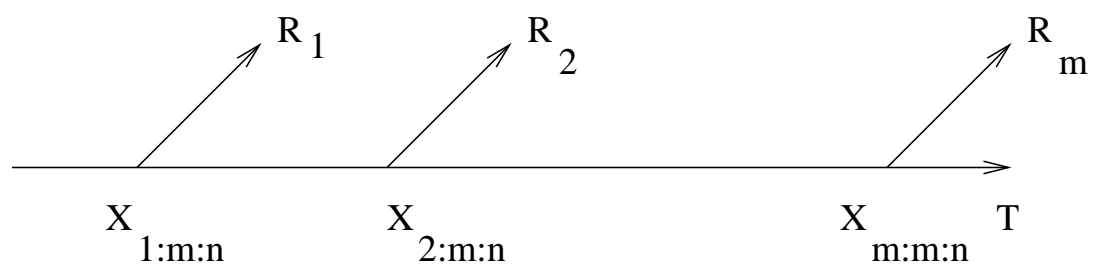

Case I

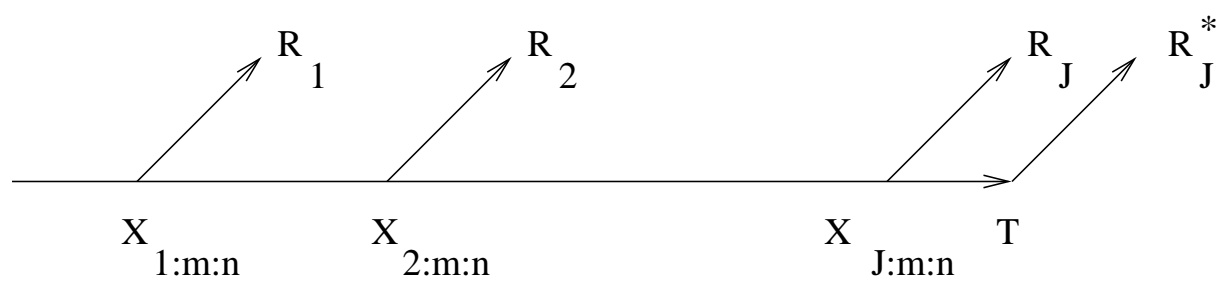

Case II

Figure 1: The schematic representation of Case I and Case II.

Therefore, in presence of Type-II progressively hybrid censoring scheme, we have one of the following types of observations;

$$
\begin{aligned}
\text { Case I: } & \left\{X_{1: m: n}, \ldots, X_{m: m: n}\right\} ; \quad \text { if } \quad X_{m: m: n}<T, \quad \text { or } \\
\text { Case II: } & \left\{X_{1: m: n}, \ldots, X_{J: m: n}\right\} ; \quad \text { if } \quad X_{J: m: n}<T<X_{J+1: m: n} .
\end{aligned}
$$


Note that for Case II, $X_{J: m: n}<T<X_{J+1: m: n}<\ldots<X_{m: m: n}$ and $X_{J+1: m: n}, \ldots, X_{m: m: n}$ are not observed.

The conventional Type-I progressive censoring scheme needs the pre-specification of $R_{1}, \ldots, R_{m}$ and also $T_{1}, \ldots, T_{m}$, see Cohen $[4,5]$ for details. The choices of $T_{1}, \ldots, T_{m}$ are not trivial. For the conventional Type-II progressive censoring scheme the experimental time is unbounded. In our proposed censoring scheme, the choice of $T$ depends how much maximum experimental time the experimenter can afford to continue. Moreover, the experimental time is bounded.

Before progressing further, we introduce/ review the following notations;

$$
\begin{aligned}
X_{i}: & \text { lifetime of the } i-t h \text { item; } i=1, \ldots, n . \\
X_{i: m: n}: & \quad i-t h \text { observed failure time } \\
f(.): & \text { probability density function (PDF) of } X_{i} \\
F(.): & \text { cumulative distribution function (CDF) of } X_{i} \\
m: & \text { the number of failures observed before termination for Case I } \\
J: & \text { the number of failures observed before termination for Case II } \\
D: & \text { the number of failures, i.e. } D=\text { m for Case I and } D=J \text { for Case II } \\
R_{i}: & \text { the number of units removed at the time of } i-t h \text { failure. } \\
R_{J}^{*}: & \text { the number of remaining units left at the time point } T \text { for Case II. } \\
\exp (\lambda): & \text { exponential random variable with PDF: } \quad \lambda e^{-\lambda x} \\
\operatorname{gamma}(\alpha, \lambda): & \text { gamma random variable with PDF: } \quad \frac{\lambda^{\alpha}}{\Gamma(\alpha)} x^{\alpha-1} e^{-\lambda x}
\end{aligned}
$$

Now we provide the MLE of $\lambda$ under the assumption that the lifetime distributions of $X_{1}, \ldots, X_{n}$ are i.i.d. $\exp (\lambda)$. 


\subsection{Maximum Likelihood Estimator}

Based on the observations (1) or (2), the log-likelihood function (without the constant term) can be written as;

$$
\begin{array}{ll}
\text { Case I: } & L(\lambda)=m \ln \lambda-\lambda \sum_{i=1}^{m}\left(1+R_{i}\right) x_{i: m: n}, \\
\text { Case II: } & L(\lambda)=J \ln \lambda-\lambda \sum_{i=1}^{J}\left(1+R_{i}\right) x_{i: m: n}-\lambda T R_{J}^{*} .
\end{array}
$$

Note that (3) and (4) can be combined as follows;

$$
L(\lambda)=D \ln \lambda-W \lambda
$$

where $D=m, W=\sum_{i=1}^{m}\left(1+R_{i}\right) x_{i: m: n}$ for Case I and $D=J, W=\sum_{i=1}^{J}\left(1+R_{i}\right) x_{i: m: n}+T R_{J}^{*}$ for Case II. Note that, for Case I, $\sum_{i=1}^{m}\left(1+R_{i}\right) x_{i: m: n}$ is a minimal sufficient statistic and for Case II, $\left(J, \sum_{i=1}^{J}\left(1+R_{i}\right) x_{i: m: n}+T R_{J}^{*}\right)$ is a joint minimal sufficient statistic. From (5), it is clear that the MLE of $\lambda$ always exists and it is

$$
\hat{\lambda}=\frac{D}{W}
$$

It is not possible to obtain the exact distribution of $\hat{\lambda}$ because of the complicated nature of the conditional distributions of $X_{1: m: n}, \ldots, X_{m: m: n}$ given $X_{m: m: n}<T$. Interestingly, the distribution of $\hat{\lambda}$ is a mixture of discrete and continuous distributions. It has a positive mass at the point 0 and it has the bounded support. Since, the exact distribution of $\hat{\lambda}$ is not known, the exact confidence interval also can not be obtained.

\section{Confidence Intervals}

In this section, we propose different confidence intervals of the unknown parameter of $\lambda$ for $D>0$. It is very difficult to obtain the confidence interval of $\lambda$ for $D=0$ and it is not pursued here. 


\subsection{Asymptotic Confidence Interval}

First let us obtain the Fisher information $I(\lambda)$, i.e.,

$$
I(\lambda)=-E\left[\frac{\partial^{2}}{\partial \lambda^{2}} L(\lambda)\right] .
$$

From (5) it follows that

$$
I(\lambda)=\frac{E(D)}{\lambda^{2}} .
$$

Observe that

$$
E(D)=\sum_{i=1}^{m} P\left(X_{i: m: n}<T\right) .
$$

For proof of (9) see in the appendix. It is not easy to compute $P\left(X_{i: m: n}<T\right)$ for general $i$, because $X_{i: m: n}$ is a sum of $i$ independent but not identically distributed exponential random variables. Therefore, for $D>0$, we propose the following approximate $100(1-\alpha) \%$ confidence interval for $\lambda$,

$$
\hat{\lambda} \pm z_{\frac{\alpha}{2}} \sqrt{\frac{\hat{\lambda}^{2}}{D}} .
$$

Meeker and Escobar [17] reported that the confidence interval based on the asymptotic theory of $\ln (\hat{\lambda})$ is superior to one of $\hat{\lambda}$. For $D>0$, the approximate $100(1-\alpha) \%$ confidence interval for $\ln (\lambda)$ is

$$
\ln (\hat{\lambda}) \pm z_{\frac{\alpha}{2}} \frac{1}{\sqrt{D}} .
$$

Therefore, for $D>0$, approximate 100(1- $\alpha) \%$ confidence interval for $\lambda$ becomes,

$$
e^{\ln (\hat{\lambda}) \pm z_{\frac{\alpha}{2}} \frac{1}{\sqrt{D}}}=\hat{\lambda} e^{ \pm z_{\frac{\alpha}{2}} \frac{1}{\sqrt{D}}}
$$

\subsection{Confidence Interval Based on Likelihood Ratio Test}

It is observed (see Meeker and Escober [17]) that the confidence interval based on the likelihood ratio test is often superior than the confidence interval based on the asymptotic 
distribution of the MLE. In this subsection we propose the confidence interval based on the likelihood ratio test, which was originally proposed by Lawless [16] for constructing confidence interval for the gamma parameters. First consider the following testing of hypothesis problem:

$$
\text { Test: } H_{0}: \lambda=\lambda_{0} \quad \text { vs. } H_{1}: \lambda \neq \lambda_{0} \text {. }
$$

The likelihood ratio (LR) statistic is:

$$
\Lambda=-2\left[L\left(\lambda_{0}\right)-L(\hat{\lambda})\right]
$$

where $L($.$) is same as defined in (5). For large sample sizes, it is possible to approximate the$ distribution $\Lambda$ under $H_{0}$ as $\chi^{2}$ with one degree of freedom. Since $\lambda$ is the scale parameter, the exact distribution of the $\Lambda$ is independent of $\lambda_{0}$. Therefore, 100(1- $\left.\alpha\right) \%$ confidence interval, $S(\lambda)$, of $\lambda$ can be constructed as;

$$
S(\lambda)=\left\{\lambda: D \ln \lambda-W \lambda \geq L(\hat{\lambda})+\frac{1}{2} \chi_{1,1-\alpha}^{2}\right\},
$$

where $\chi_{1,1-\alpha}^{2}$ is the upper $100(1-\alpha) \%$ percentile point of the $\chi^{2}$ distribution with one degree of freedom. Since $(D \ln \lambda-W \lambda)$ is a unimodal function, therefore, for a given $\alpha, S(\lambda)$ is a unique interval.

\subsection{Bootstrap Confidence Intervals}

In this subsection we propose two confidence intervals based on bootstrapping. The two bootstrap methods that are widely used in practice are;

(1) The percentile bootstrap (Boot-p) proposed by Efron [8], and

(2) The bootstrap-t method (Boot-t) proposed by Hall [13].

It is observed that in this type of situations (Kundu, Kannan and Balakrishnan, [15]), the non-parametric bootstrap method does not work well. We propose the following two parametric bootstrap confidence intervals for $\lambda$. 
[1] Estimate $\lambda$, say $\hat{\lambda}$, from the sample using (6).

[2] Generate a bootstrap sample $\left\{X_{1: m: n}^{*}, \ldots, X_{D^{*}: m: n}^{*}\right\}$, using $\hat{\lambda}, R_{1}, \ldots, R_{m}$ and $T$. Obtain the bootstrap estimate of $\lambda$, say $\hat{\lambda}^{*}$ using the bootstrap sample.

[3] Repeat Step [2] NBOOT times.

[4] Let $\widehat{C D F}(x)=P\left(\hat{\lambda}^{*} \leq x\right)$, be the cumulative distribution function of $\hat{\lambda}^{*}$. Define $\hat{\lambda}_{\text {Boot-p }}(x)=\widehat{C D F}{ }^{-1}(x)$ for a given $x$. The approximate 100(1- $\left.\alpha\right) \%$ confidence interval for $\lambda$ is given by

$$
\left(\hat{\lambda}_{\text {Boot-p }}\left(\frac{\alpha}{2}\right), \hat{\lambda}_{\text {Boot-p }}\left(1-\frac{\alpha}{2}\right)\right)
$$

\section{Boot-т Method:}

[1] Estimate $\lambda$, say $\hat{\lambda}$, from the sample using (6) as before.

[2] Generate a bootstrap sample $\left\{X_{1: m: n}^{*}, \ldots, X_{D^{*}: m: n}^{*}\right\}$, using $\hat{\lambda}, R_{1}, \ldots, R_{m}, T$ and obtain $\hat{\lambda}^{*}$. Also compute $\hat{V}\left(\hat{\lambda}^{*}\right)=\frac{\hat{\lambda}^{*}}{D^{*}}$ for $D^{*}>0$.

[3] Determine the $T^{*}$ statistic

$$
T^{*}=\frac{\left(\hat{\lambda}^{*}-\hat{\lambda}\right)}{\sqrt{\hat{V}\left(\hat{\lambda}^{*}\right)}} .
$$

[4] Repeat Step [2] - [3] NBOOT times.

[5] Let $\widehat{C D F}(x)=P\left(T^{*} \leq x\right)$, be the cumulative distribution function of $T^{*}$. For a given $x$, define $\hat{\lambda}_{\text {Boot-t }}(x)=\hat{\lambda}+\sqrt{\hat{V}\left(\hat{\lambda}^{*}\right)} \widehat{C D F}{ }^{-1}(x)$. The approximate $100(1-\alpha) \%$ confidence interval for $\lambda$ is given by

$$
\left(\hat{\lambda}_{\text {Boot-t }}\left(\frac{\alpha}{2}\right), \hat{\lambda}_{\text {Boot-t }}\left(1-\frac{\alpha}{2}\right)\right) .
$$




\section{Bayesian Analysis}

In this section we approach the problem from the Bayesian point of view. In the context of exponential lifetimes $\lambda$ may be reasonably modeled by the gamma priors. We assume that $\lambda$ is distributed as gamma $(a, b)$, where the gamma parameters $a$ and $b$ are assumed to be positive. When $a=b=0$, it is the non-informative prior of $\lambda$. The posterior density of $\lambda$ given the data can be written as

$$
l(\lambda \mid d a t a) \propto \lambda^{D+a-1} e^{-\lambda(W+b)} .
$$

From (16) it is clear that the posterior distribution of $\lambda$ is also $\operatorname{gamma}(D+a, W+b)$. Therefore, the Bayes estimate of $\lambda$ under squared error loss function is

$$
\hat{\lambda}_{\text {Bayes }}=\frac{D+a}{W+b}
$$

Interestingly, when $a=b=0$, the Bayes estimator coincides with the MLE.

The credible interval of $\lambda$ can be obtained using the posterior distribution of $\lambda$. Note that a posteriori

$$
Z=2 \lambda(W+b)
$$

follows $\chi^{2}$ distribution with $2(D+a)$ degrees of freedom provided $2(D+a)$ is a positive integer. Therefore, $100(1-\alpha) \%$ credible interval for $\lambda$ can be obtained as

$$
\left[\frac{\chi_{2(D+a), 1-\frac{\alpha}{2}}^{2}}{2(W+b)}, \frac{\chi_{2(D+a), \frac{\alpha}{2}}^{2}}{2(W+b)}\right]
$$

where $\chi_{2(D+a), \frac{\alpha}{2}}^{2}$ and $\chi_{2(D+a), 1-\frac{\alpha}{2}}^{2}$ denote the lower and upper $\frac{\alpha}{2}$-th percentile points of a $\chi^{2}$ distribution with $2(D+a)$ degrees of freedom. Note that if $2(D+a)$ is not an integer then gamma distribution can be used to construct the credible interval. If no prior information is available, then non-informative priors can be used to compute the credible interval for $\lambda$. Alternatively, using the suggestion of Congdon [6], very small positive values of $a$ and $b$ can be used to construct the Bayes estimate and the corresponding credible interval. 


\section{Numerical Results and Discussions}

Since the performance of the different methods can not compared theoretically, we use Monte Carlo simulations to compare different methods for different parameter values and for different sampling schemes. The term different sampling schemes means for different sets of $R_{i}$ 's and for different $T$ values. All the computations are performed using Pentium IV processor and using the random number generation algorithm RAN2 of Press et al. [18]. All the programs are written in FORTRAN and they can be obtained from the authors on request.

Before progressing further, first we describe how we generate Type-II progressively hybrid censored data for a given set $n, m, R_{1}, \ldots, R_{m}$ and $T$. We use the following transformation suggested in Balakrishnan and Aggarwala [1].

$$
\begin{aligned}
Z_{1} & =n X_{1: m: n} \\
Z_{2} & =\left(n-R_{1}-1\right)\left(X_{2: m: n}-X_{1: m: n}\right) \\
& \vdots \\
Z_{m} & =\left(n-R_{1}-\ldots-R_{m-1}-m+1\right)\left(X_{m: m: n}-X_{m-1: m: n}\right) .
\end{aligned}
$$

It is known that if $X_{i}$ 's are i.i.d $\exp (\lambda)$, then the spacings $Z_{i}$ 's are also $i . i . d \exp (\lambda)$ random variables. From (19) it follows that

$$
\begin{aligned}
X_{1: m: n} & =\frac{1}{n} Z_{1} \\
X_{2: m: n} & =\frac{1}{n-R_{1}-1} Z_{2}+\frac{1}{n} Z_{1} \\
& \vdots \\
X_{m: m: n} & =\frac{1}{n-R_{1}-\ldots-R_{m-1}-m+1} Z_{m}+\ldots+\frac{1}{n} Z_{1} .
\end{aligned}
$$

Using (20), Type-II progressively hybrid censored data can be easily generated as follows. For a given $n, m, R_{1}, \ldots, R_{m}$, we generate $X_{1: m: n}, \ldots, X_{m: m: n}$ using (20). If $X_{m: m: n}<T$, then we have Case I and the corresponding sample is $\left\{\left(X_{1: m: n}, R_{1}\right), \ldots,\left(X_{m: m: n}, R_{m}\right)\right\}$. If 
$X_{m: m: n}>T$, then we have Case II and we find $J$, such that $X_{J: m: n}<T<X_{J+1: m: n}$. The corresponding Type-II hybrid censored sample is $\left\{\left(X_{1: m: n}, R_{1}\right), \ldots,\left(X_{J: m: n}, R_{J}\right)\right\}$ and $R_{J}^{*}$, where $R_{J}^{*}$ is same as defined before.

We consider different $n, m$ and $T$. We have used three different sampling schemes, namely: Scheme 1: $R_{1}=\ldots=R_{m-1}=0$ and $R_{m}=n-m$. Scheme 2: $R_{1}=n-m$ and $R_{2}=\ldots=R_{m}=0$. Scheme $3: R_{1}=\ldots=R_{m-1}=1$ and $R_{m}=n-2 m+1$. Without loss of generality we take $\lambda=1$ in each case. We compute the MLE and the $95 \%$ confidence intervals using (I) asymptotic distribution of $\hat{\lambda}$, (II) Boot-p method, (III) Boot-t method, (IV) asymptotic distribution of $\ln \hat{\lambda}$, (V) likelihood ratio method, when $D>0$. For comparison purposes we also compute the $95 \%$ Bayes credible intervals using the noninformative priors, namely $a_{1}=a_{2}=b_{1}=b_{2}=0$. We replicate the process 1000 times in each case and report the average biases, mean squared errors (MSEs) and the coverage probabilities. In any replication if $D=0$, we have not considered that sample. The average biases of the MLEs and the corresponding MSEs are reported in Table 1. The average confidence/ credible lengths and the corresponding coverage percentages are reported in the Tables 2-7.

From Table 1, the following observations are made. For fixed $n$ as $m$ increases the biases and the MSEs decrease for all cases as expected. For fixed $m$ as $n$ increases although MSEs decrease but the biases increase. The later phenomenon is quite counter intuitive. It is also observed that the MLEs for schemes 1 and 3 behave quite similarly in terms of biases and MSEs unless both $n$ and $m$ are small or $T$ is small. The MLEs for scheme 2 have smaller biases than the corresponding MLEs for the other two schemes unless $T$ is very small, although the MSEs are always larger.

Now we compare different confidence/ credible intervals in terms of their average lengths and coverage probabilities. In general it is observed that most of the methods work well 
unless $T$ is very small or $n$ and $m$ are very small. For most of the methods it is observed that the average confidence/ credible lengths decrease as $n$ increases for fixed $m$ or the other way. For most of the methods, scheme 1 and scheme 3 behave very similarly although the confidence intervals for scheme 1 are usually slightly shorter than scheme 3 . The confidence intervals for scheme 2 are usually larger than the other two.

It is observed that the confidence intervals based on MLEs work quite well unless $T, m$ and $n$ are very small. In that case it can not maintain the nominal level at least for schemes 1 and 3. Interestingly, even when $m, n$ and $T$ are small it works quite well for scheme 2 in terms of the coverage probability although the confidence lengths are significantly larger. In general Boot-p confidence intervals do not work that well. It can not maintain the nominal level even when $T$ is large. In fact, the average confidence length are usually larger than the asymptotic confidence lengths unless $\mathrm{n}$ and $\mathrm{m}$ are very small. Boot-t confidence intervals work definitely better than the Boot-p confidence intervals both in terms of coverage percentages and confidence lengths except scheme 2. The average confidence lengths for Boot-t confidence intervals are slightly smaller than the asymptotic confidence intervals at least for small sample sizes, but the coverage probabilities are also slightly lower than the nominal level in general. The confidence intervals based on the asymptotic distribution of $\ln \hat{\lambda}$ also work quite well for all sample sizes and for all the schemes. In most of the cases the coverage probabilities are quite close to the nominal level, although the average confidence lengths are significantly larger than the asymptotic confidence intervals based on MLEs or Boot-t confidence intervals. The confidence intervals based on the likelihood ratio works very well even for small $n, m$ and $T$ and also for all the schemes. It can maintain the coverage percentages to the nominal level in most of the cases. The average confidence lengths are larger than the asymptotic confidence intervals based on MLEs or Boot-t confidence intervals but smaller than the confidence intervals based on the asymptotic distribution of $\ln \hat{\lambda}$. The Bayes credible intervals with respect to the non-informative priors works also work very 
well. It can maintain the coverage probabilities in most of the cases considered. The average credible lengths are also slightly smaller than the likelihood ratio based confidence intervals. Comparing all the methods, we suggest that likelihood ratio based confidence intervals or Bayes credible intervals can be used for practical purposes.

We compare different sampling schemes with respect to their information measures. Other than sampling schemes 1,2 and 3 we also consider the following schemes:

Scheme 4: $R_{1}=\ldots=R_{m-1}=2$ and $R_{m}=n-3 m+2$.

Scheme 5: $R_{1}=\ldots=R_{m-1}=3$ and $R_{m}=n-4 m+3$.

Scheme 6: $R_{1}=\ldots=R_{m-1}=4$ and $R_{m}=n-5 m+4$.

Scheme 7: $R_{1}=\ldots=R_{m-1}=5$ and $R_{m}=n-6 m+5$.

Scheme 8: $R_{1}=\ldots=R_{m-1}=6$ and $R_{m}=n-7 m+6$.

We already observed that the Fisher Information of $\lambda$ in (8) where $E(D)$ can be calculated using (9). Since it is not very easy to compute (9), we use Monte Carlo simulation techniques to compute the value of $E(D)$ for different sampling schemes and for different $T$ values. We have generated $X_{i: m: n}$ using the transformation (20) and compute the $P\left(X_{i: m: n}<T\right)$ based on 1000 replications. The results are reported in the Table 8. Interestingly it is observed that scheme 1 has uniformly maximum information of $\lambda$ and scheme 2 has the lowest one. The other sampling schemes have the information in between these two. Moreover, from Table 8 , we also obtain the average duration of the experiments and the probability of termination due to case 1. It is observed, although not very surprising, that the average duration of the experiment is smallest for scheme 1 and highest for scheme 2 and the probability of termination due to case 1 is highest for scheme 1 and lowest for scheme 2. Other schemes all lie between these two extreme schemes. Therefore, for an experimenter, it might be necessary to choose a balance between the expected number of failures $(E(D))$ and the expected duration of the experiment and appropriately choose the scheme. For example, let $c_{1}$ be the cost incurred due to a failed unit and $c_{2}$ be the cost incurred due to unit time of the 
experiment. Then the average cost of an experiment for a particular scheme is $c_{1} N+c_{2} S$, where $N$ denotes the expected number of failures and $S$ denotes the expected duration of the experiment for that particular scheme. The experimenter might be interested in choosing that particular scheme which minimizes the expected cost $c_{1} N+c_{2} S$. For that purpose, the Table 8 will be useful. Note that although we have reported the result for $\lambda=1$, but since it is the scale parameter, it can be used for other $\lambda$ 's also, with the appropriate changes in $T$.

\section{Data Analysis}

In this section we consider one real-life data analysis from Lawless [16]. It consists of failure or censoring times for 36 appliances subjected to an automatic life test. The data are given below, consisting of only the failure times. From the given data we created two different artificial Type II progressively hybrid censored data.

DATA SET:- 11, 35, 49, 170, 329, 381, 708, 958, 1062, 1167, 1594, 1925, 1990, 2223, 2327, 2400, 2451, 2471, 2551, 2565, 2568, 2694, 2702, 2761, 2831, 3034, 3059, 3112, 3214, 3478, 3504, 4329, 6367, 6976, 7846, 13403.

ExAmple 1: In this case $n=36$ and we take $m=10, T=2600, R_{1}=R_{2}=\ldots=R_{9}=2$, $R_{10}=8$. Thus the Type II progressively hybrid censored sample is : $11,35,49,170,329$, 958, 1925, 2223, 2400, 2568. From the above sample data, we obtain $D=m=10$ and

$$
W=\sum_{i=1}^{10}\left(1+R_{i}\right) x_{i: m: n}=47412,
$$

which yields

$$
\hat{\lambda}=\frac{10}{47412}=2.10917 \times 10^{-4} .
$$

Using the properties of the Fisher Information Matrix, we obtain $\operatorname{var}(\hat{\lambda}) \approx \frac{\hat{\lambda}^{2}}{D}$ and hence $95 \%$ asymptotic confidence interval for $\lambda$ based on $\hat{\lambda}, \ln \hat{\lambda}$ and confidence interval based on 
likelihood ratio test are

$$
\begin{aligned}
& \left(0.80189 \times 10^{-4}, 3.41645 \times 10^{-4}\right), \\
& \left(1.13484 \times 10^{-4}, 3.92004 \times 10^{-4}\right), \\
& \left(1.05731 \times 10^{-4}, 3.66227 \times 10^{-4}\right)
\end{aligned}
$$

respectively.

The corresponding Boot-t, Boot-p confidence intervals are

$$
\left(0.53967 \times 10^{-4}, 3.21717 \times 10^{-4}\right), \quad\left(1.17794 \times 10^{-4}, 3.97859 \times 10^{-4}\right)
$$

respectively and the $95 \%$ Bayes credible interval becomes;

$$
\left[\frac{\chi_{20,0.975}^{2}}{2 \times 47412}, \frac{\chi_{20,0.025}^{2}}{2 \times 47412}\right]=\left(1.01143 \times 10^{-4}, 3.60347 \times 10^{-4}\right) .
$$

Example 2: Now consider $m=10$ and $T=2000$ and $R_{i}$ 's are same as before. In this case the progressively hybrid censored sample obtained as : 11, 35, 49, 170, 329, 958, 1925. From the above sample data, we obtain the following: $D=J=7$, also

$$
W=\sum_{i=1}^{7}\left(1+R_{i}\right) x_{i: m: n}=10431
$$

which yields

$$
\hat{\lambda}=\frac{7}{10431+15 \times 2000}=1.73134 \times 10^{-4} .
$$

Therefore, $95 \%$ asymptotic confidence interval based on $\hat{\lambda}, \ln \hat{\lambda}$, confidence interval based on likelihood ratio test, Boot-t and Boot-p methods for $\lambda$ are

$$
\begin{array}{cc}
\left(0.44875 \times 10^{-4},\right. & \left.3.01393 \times 10^{-4}\right), \\
\left(0.82538 \times 10^{-4},\right. & \left.3.63173 \times 10^{-4}\right), \\
\left(0.74782 \times 10^{-4},\right. & \left.3.33374 \times 10^{-4}\right), \\
\left(-0.11094 \times 10^{-4},\right. & \left.2.88180 \times 10^{-4}\right), \\
\left(0.81981 \times 10^{-4},\right. & \left.3.26588 \times 10^{-4}\right),
\end{array}
$$


respectively. The $95 \%$ Bayes credible interval becomes;

$$
\left[\frac{\chi_{14,0.975}^{2}}{2 \times(10431+30000)}, \frac{\chi_{14,0.025}^{2}}{2 \times(10431+30000)}\right]=\left[0.69608 \times 10^{-4}, 3.23007 \times 10^{-4}\right] .
$$

\section{Conclusions}

In this paper we discuss a new censoring scheme namely the Type II progressively hybrid censoring scheme. Assuming that the lifetime distributions are exponentially distributed we obtain the maximum likelihood estimators of the unknown parameter and propose different confidence intervals using asymptotic distributions as well as using bootstrap methods. Bayesian estimate of the unknown parameter is also proposed and it is observed that the Bayes estimate with respect to non-informative prior works quite well in this case. Although we have assumed that the lifetime distributions are exponential but most of the methods can be extended for other distributions also, like Weibull distribution or gamma distribution. The work is in progress and it will be reported elsewhere.

\section{ACKNOWLEDGMENTS:}

The authors are thankful to two referees for their very helpful comments. The authors are also very thankful to Asia-Associate editor Professor J.C. Lee for his encouragements.

\section{APPENDIX}

Proof of (9): Observe that for $1 \leq i \leq m-1$,

$$
\begin{aligned}
P\left(X_{i: m: n}<T\right) & =P\left(X_{i: m: n}<T, X_{i+1: m: n}<T\right)+P\left(x_{i: m: n}<T, X_{i+1: m: n} \geq T\right) \\
& =P\left(X_{i+1: m: n}<T\right)+P(D=i)
\end{aligned}
$$




$$
\begin{aligned}
& =P\left(X_{m: m: n}<T\right)+\sum_{k=i}^{m-1} P(D=k) \\
& =\sum_{k=i}^{m} P(D=k) .
\end{aligned}
$$

Therefore,

$$
\sum_{i=1}^{m} P\left(X_{i: m: n}<T\right)=\sum_{i=1}^{m} \sum_{k=i}^{m} P(D=k)=\sum_{k=1}^{m} \sum_{i=1}^{k} P(D=k)=\sum_{k=1}^{m} k P(D=k)=E(D) .
$$

\section{References}

[1] Balakrishnan, N. and Aggarwala, R. (2000), Progressive Censoring: Theory, Methods and Applications, Boston: Birkhäuser.

[2] Chen, S.M. and Bhattacharya, G.K. (1988), "Exact confidence bounds for an exponential parameter hybrid censoring", Communications in Statistics - Theory and Methods, vol. 17, 1858 - 1870.

[3] Childs, A., Chandrasekhar, B., Balakrishnan, N. and Kundu, D. (2003), "Exact Likelihood Inference Based on Type-I and Type-II Hybrid Censored Samples from the Exponential Distribution", Annals of the Institute of Statistical Mathematics, vol. 55, 319-330.

[4] Cohen, A.C. (1963), "Progressively censored samples in life testing", Technometrics, vol. 5, $327-329$.

[5] Cohen, A.C. (1966), "Life-testing and early failure", Technometrics, vol. 8, 539 549.

[6] Congdon, P. (2001), Bayesian Statistical Modeling, John Wiley, New York. 
[7] Draper, N. and Guttman, T. (1987), "Bayesian analysis of hybrid life-test with exponential failure times", Annals of the Institute of Statistical Mathematics, vol. $39,219-255$.

[8] Efron, B. (1982), The Jackknife, the Bootstrap and Other Re-sampling Plans, CBMS-NSF Regional Conference Series in Applied Mathematics, vol. 38, SIAM, Philadelphia, PA.

[9] Epstein B. (1954), "Truncated life-tests in the exponential case", Annals of Mathematical Statistics, vol. 25, 555 - 564.

[10] Epstein, B. (1960), "Estimation from life-test data", Technometrics, vol. 2, 447 454.

[11] Fairbanks, K., Madasan, R. and Dykstra, R. (1982), "A confidence interval for an exponential parameter from hybrid life-test", Journal of the American Statistical Association, vol. 77, 137 - 140 .

[12] Gupta, R.D. and Kundu, D. (1998), "Hybrid censoring schemes with exponential failure distribution", Communications in Statistics - Theory and Methods, vol. 27, $3065-3083$.

[13] Hall, P. (1988), "Theoretical comparison of bootstrap confidence intervals", Annals of Statistics, vol. 16, 927 - 953.

[14] Jeong, H.S., Park, J.I. and Yum, B.J. (1996), "Development of $(r, T)$ hybrid sampling plans for exponential lifetime distributions", Journal of Applied Statistics, vol. 23, 601-607. 
[15] Kundu, D., Kannan, N. and Balakrishnan, N. (2004), "Analysis of progressively censored competing risks data", Handbook of Statistics, vol. 23, eds., Balakrishnan, N. and Rao, C.R., Elsevier, New York.

[16] Lawless, J.F. (1982), Statistical Models and Methods for Lifetime Data, Wiley, New York.

[17] Meeker, W.Q. and Escobar, L.A. (1998), Statistical Methods for Reliability Data, Wiley, New York.

[18] Press, W.H., Flannery, B.P., Teukolsky, S.A. and Vetterling, W.T. (1991), Numerical Recipes: The Art of Scientific Computing, Cambridge University Press, Cambridge, U.K. 
Table 1: The biases and MSEs of the MLEs for different sample sizes and for different schemes are presented. Each value represents the average bias and the corresponding MSE is reported within bracket.

\begin{tabular}{|l|l|l|l|l|l|}
\hline$(n, m)$ & Scheme & $\mathrm{T}=0.25$ & $\mathrm{~T}=0.50$ & $\mathrm{~T}=1.00$ & $\mathrm{~T}=2.00$ \\
& & & & & \\
\hline$(15,5)$ & 1 & $0.1824(0.8036)$ & $0.2851(0.7217)$ & $0.3075(0.6878)$ & $0.3076(0.6877)$ \\
& 2 & $0.3001(1.0138)$ & $0.2511(0.8443)$ & $0.2373(0.7430)$ & $0.2771(0.7153)$ \\
& 3 & $0.1800(0.7928)$ & $0.2696(0.7356)$ & $0.3057(0.6910)$ & $0.3076(0.6877)$ \\
\hline$(25,5)$ & 1 & $0.2702(0.7396)$ & $0.3063(0.6901)$ & $0.3076(0.6877)$ & $0.3076(0.6877)$ \\
& 2 & $0.3096(0.9855)$ & $0.2579(0.8392)$ & $0.2369(0.7406)$ & $0.2773(0.7152)$ \\
& 3 & $0.2627(0.7469)$ & $0.3058(0.6910)$ & $0.3076(0.6877)$ & $0.3076(0.6877)$ \\
\hline$(50,5)$ & 1 & $0.3071(0.6886)$ & $0.3076(0.6877)$ & $0.3076(0.6877)$ & $0.3076(0.6877)$ \\
& 2 & $0.3133(0.9814)$ & $0.2627(0.8356)$ & $0.2413(0.7356)$ & $0.2778(0.7145)$ \\
& 3 & $0.3063(0.6901)$ & $0.3076(0.6877)$ & $0.3076(0.6877)$ & $0.3076(0.6877)$ \\
\hline$(100,5)$ & 1 & $0.3076(0.6877)$ & $0.3076(0.6877)$ & $0.3076(0.6877)$ & $0.3076(0.6877)$ \\
& 2 & $0.3170(0.9780)$ & $0.2627(0.8322)$ & $0.2425(0.7334)$ & $0.2787(0.7140)$ \\
& 3 & $0.3076(0.6877)$ & $0.3076(0.6877)$ & $0.3076(0.6877)$ & $0.3076(0.6877)$ \\
\hline$(25,10)$ & 1 & $0.0281(0.2152)$ & $0.0813(0.1767)$ & $0.1099(0.1558)$ & $0.1100(0.1557)$ \\
& 2 & $0.0957(0.4378)$ & $0.0857(0.2920)$ & $0.0788(0.1976)$ & $0.0931(0.1679)$ \\
& 3 & $0.0520(0.2433)$ & $0.0672(0.1781)$ & $0.1048(0.1604)$ & $0.1100(0.1557)$ \\
\hline$(50,10)$ & 1 & $0.0901(0.1726)$ & $0.1100(0.1557)$ & $0.1100(0.1557)$ & $0.1100(0.1557)$ \\
& 2 & $0.1049(0.4230)$ & $0.0888(0.2862)$ & $0.0783(0.1968)$ & $0.0925(0.1679)$ \\
& 3 & $0.0813(0.1767)$ & $0.1099(0.1558)$ & $0.1100(0.1557)$ & $0.1100(0.1557)$ \\
\hline$(100,10)$ & 1 & $0.1100(0.1557)$ & $0.1100(0.1557)$ & $0.1100(0.1557)$ & $0.1100(0.1557)$ \\
& 2 & $0.1088(0.4171)$ & $0.0899(0.2845)$ & $0.0800(0.1977)$ & $0.0926(0.1675)$ \\
& 3 & $0.1100(0.1557)$ & $0.1100(0.1557)$ & $0.1100(0.1557)$ & $0.1100(0.1557)$ \\
\hline$(50,15)$ & 1 & $0.0139(0.1075)$ & $0.0628(0.0864)$ & $0.0654(0.0840)$ & $0.0654(0.0840)$ \\
& 2 & $0.0365(0.2689)$ & $0.0349(0.1723)$ & $0.0392(0.1188)$ & $0.0496(0.0928)$ \\
& 3 & $0.0133(0.1101)$ & $0.0558(0.0901)$ & $0.0654(0.0840)$ & $0.0654(0.0840)$ \\
\hline & 1 & $0.0643(0.0851)$ & $0.0654(0.0840)$ & $0.0654(0.0840)$ & $0.0654(0.0840)$ \\
& 3 & $0.0385(0.2615)$ & $0.0353(0.1716)$ & $0.0388(0.1184)$ & $0.0494(0.0927)$ \\
& $0.0628(0.0864)$ & $0.0654(0.0840)$ & $0.0654(0.0840)$ & $0.0654(0.0840)$ \\
\hline
\end{tabular}


Table 2: The average confidence lengths and the corresponding coverage percentages of the MLEs for different sample sizes and for different schemes are presented. Each value represents the average confidence length and the corresponding coverage percentage is reported within bracket.

\begin{tabular}{|l|l|l|l|l|l|}
\hline$(n, m)$ & Scheme & $\mathrm{T}=0.25$ & $\mathrm{~T}=0.50$ & $\mathrm{~T}=1.00$ & $\mathrm{~T}=2.00$ \\
& & & & & \\
\hline$(15,5)$ & 1 & $2.4017(0.88)$ & $2.2913(0.91)$ & $2.2923(0.96)$ & $2.2923(0.96)$ \\
& 2 & $3.6823(0.95)$ & $2.9609(0.94)$ & $2.5021(0.93)$ & $2.3239(0.95)$ \\
& 3 & $2.5006(0.87)$ & $2.3065(0.95)$ & $2.2917(0.96)$ & $2.2923(0.96)$ \\
\hline$(25,5)$ & 1 & $2.2931(0.95)$ & $2.2913(0.96)$ & $2.2923(0.96)$ & $2.2923(0.96)$ \\
& 2 & $3.6512(0.95)$ & $2.9454(0.94)$ & $2.4936(0.93)$ & $2.3223(0.95)$ \\
& 3 & $2.3029(0.93)$ & $2.2915(0.96)$ & $2.2923(0.96)$ & $2.2923(0.96)$ \\
\hline$(50,5)$ & 1 & $2.2923(0.96)$ & $2.2923(0.96)$ & $2.2923(0.96)$ & $2.2923(0.96)$ \\
& 2 & $3.6172(0.94)$ & $2.9342(0.94)$ & $2.4927(0.94)$ & $2.3216(0.95)$ \\
& 3 & $2.2913(0.96)$ & $2.2923(0.96)$ & $2.2923(0.96)$ & $2.2923(0.96)$ \\
\hline$(100,5)$ & 1 & $2.2923(0.96)$ & $2.2923(0.96)$ & $2.2923(0.96)$ & $2.2923(0.96)$ \\
& 2 & $3.6039(0.94)$ & $2.9263(0.94)$ & $2.4913(0.94)$ & $2.3218(0.95)$ \\
& 3 & $2.2923(0.96)$ & $2.2923(0.96)$ & $2.2923(0.96)$ & $2.2923(0.96)$ \\
\hline$(25,10)$ & 1 & $1.6678(0.93)$ & $1.3934(0.93)$ & $1.3759(0.96)$ & $1.3760(0.96)$ \\
& 2 & $2.4892(0.91)$ & $1.9690(0.91)$ & $1.6157(0.93)$ & $1.4321(0.95)$ \\
& 3 & $1.7954(0.91)$ & $1.4661(0.94)$ & $1.3772(0.94)$ & $1.3760(0.96)$ \\
\hline$(50,10)$ & 1 & $1.3829(0.94)$ & $1.3760(0.96)$ & $1.3760(0.96)$ & $1.3760(0.96)$ \\
& 2 & $2.4567(0.91)$ & $1.9537(0.92)$ & $1.6088(0.93)$ & $1.4302(0.95)$ \\
& 3 & $1.3934(0.93)$ & $1.3759(0.96)$ & $1.3760(0.96)$ & $1.3760(0.96)$ \\
\hline$(100,10)$ & 1 & $1.3760(0.96)$ & $1.3760(0.96)$ & $1.3760(0.96)$ & $1.3760(0.96)$ \\
& 2 & $2.4404(0.91)$ & $1.9459(0.92)$ & $1.6069(0.93)$ & $1.4295(0.95)$ \\
& 3 & $1.3760(0.96)$ & $1.3760(0.96)$ & $1.3760(0.96)$ & $1.3760(0.96)$ \\
\hline$(50,15)$ & 1 & $1.1865(0.94)$ & $1.0784(0.95)$ & $1.0783(0.96)$ & $1.0783(0.96)$ \\
& 2 & $2.0074(0.91)$ & $1.5769(0.93)$ & $1.2861(0.93)$ & $1.1295(0.96)$ \\
& 3 & $1.2511(0.93)$ & $1.0840(0.95)$ & $1.0783(0.96)$ & $1.0783(0.96)$ \\
\hline$(100,15)$ & 1 & $1.0783(0.96)$ & $1.0783(0.96)$ & $1.0783(0.96)$ & $1.0783(0.96)$ \\
& 2 & $1.9889(0.91)$ & $1.5684(0.93)$ & $1.2829(0.93)$ & $1.1287(0.96)$ \\
& 3 & $1.0784(0.95)$ & $1.0783(0.96)$ & $1.0783(0.96)$ & $1.0783(0.96)$ \\
\hline
\end{tabular}


Table 3: The average confidence lengths and the corresponding coverage percentages of the Boot-P confidence intervals for different sample sizes and for different schemes are presented. Each value represents the average confidence length and the corresponding coverage percentage is reported within bracket.

\begin{tabular}{|l|l|l|l|l|l|}
\hline$(n, m)$ & Scheme & $\mathrm{T}=0.25$ & $\mathrm{~T}=0.50$ & $\mathrm{~T}=1.00$ & $\mathrm{~T}=2.00$ \\
& & & & & \\
\hline$(15,5)$ & 1 & $1.9641(0.89)$ & $2.0567(0.90)$ & $2.1639(0.91)$ & $2.1697(0.91)$ \\
& 2 & $4.0889(0.94)$ & $3.4471(0.94)$ & $3.1507(0.91)$ & $3.1103(0.90)$ \\
& 3 & $3.1890(0.88)$ & $3.1986(0.88)$ & $3.2051(0.89)$ & $3.1763(0.89)$ \\
\hline$(25,5)$ & 1 & $3.1261(0.86)$ & $3.1031(0.90)$ & $3.0749(0.90)$ & $3.0740(0.90)$ \\
& 2 & $4.0834(0.97)$ & $3.3615(0.94)$ & $3.1144(0.92)$ & $3.0763(0.90)$ \\
& 3 & $3.2049(0.86)$ & $3.2115(0.89)$ & $3.1760(0.89)$ & $3.1748(0.89)$ \\
\hline$(50,5)$ & 1 & $3.0999(0.90)$ & $3.0746(0.90)$ & $3.0740(0.90)$ & $3.0740(0.90)$ \\
& 2 & $3.9500(0.97)$ & $3.3697(0.96)$ & $3.1466(0.93)$ & $3.1046(0.91)$ \\
& 3 & $3.2040(0.89)$ & $3.1754(0.89)$ & $3.1748(0.89)$ & $3.1748(0.89)$ \\
\hline$(100,5)$ & 1 & $3.0745(0.90)$ & $3.0740(0.90)$ & $3.0740(0.90)$ & $3.0740(0.90)$ \\
& 2 & $3.9951(0.97)$ & $3.4406(0.95)$ & $3.1795(0.93)$ & $3.1508(0.91)$ \\
& 3 & $3.1752(0.89)$ & $3.1748(0.89)$ & $3.1748(0.89)$ & $3.1748(0.89)$ \\
\hline$(25,10)$ & 1 & $1.7888(0.90)$ & $1.6776(0.89)$ & $1.6206(0.92)$ & $1.6086(0.92)$ \\
& 2 & $2.5375(0.94)$ & $2.0910(0.94)$ & $1.8125(0.93)$ & $1.6932(0.92)$ \\
& 3 & $1.8996(0.91)$ & $1.6998(0.91)$ & $1.6479(0.91)$ & $1.6199(0.91)$ \\
\hline$(50,10)$ & 1 & $1.6798(0.89)$ & $1.6154(0.92)$ & $1.6086(0.92)$ & $1.6086(0.92)$ \\
& 2 & $2.5273(0.93)$ & $2.0906(0.92)$ & $1.8029(0.92)$ & $1.6804(0.92)$ \\
& 3 & $1.6930(0.90)$ & $1.6302(0.91)$ & $1.6186(0.91)$ & $1.6185(0.91)$ \\
\hline$(100,10)$ & 1 & $1.6137(0.92)$ & $1.6086(0.92)$ & $1.6086(0.92)$ & $1.6086(0.92)$ \\
& 2 & $2.4156(0.95)$ & $2.0271(0.94)$ & $1.7493(0.93)$ & $1.6359(0.92)$ \\
& 3 & $1.6245(0.91)$ & $1.6185(0.91)$ & $1.6185(0.91)$ & $1.6185(0.91)$ \\
\hline$(50,15)$ & 1 & $1.2982(0.91)$ & $1.2225(0.92)$ & $1.1915(0.93)$ & $1.1914(0.93)$ \\
& 2 & $2.0038(0.93)$ & $1.6262(0.92)$ & $1.3799(0.92)$ & $1.2579(0.93)$ \\
& 3 & $1.3327(0.93)$ & $1.2307(0.91)$ & $1.1911(0.93)$ & $1.1895(0.93)$ \\
\hline$(100,15)$ & 1 & $1.2166(0.93)$ & $1.1915(0.93)$ & $1.1914(0.93)$ & $1.1914(0.93)$ \\
& 2 & $2.0522(0.94)$ & $1.6657(0.94)$ & $1.3916(0.94)$ & $1.2542(0.93)$ \\
& 3 & $1.2202(0.92)$ & $1.1895(0.93)$ & $1.1895(0.93)$ & $1.1895(0.93)$ \\
\hline
\end{tabular}


Table 4: The average confidence lengths and the corresponding coverage percentages of the Boot-t confidence intervals for different sample sizes and for different schemes are presented. Each value represents the average confidence length and the corresponding coverage percentage is reported within bracket.

\begin{tabular}{|l|l|l|l|l|l|}
\hline$(n, m)$ & Scheme & $\mathrm{T}=0.25$ & $\mathrm{~T}=0.50$ & $\mathrm{~T}=1.00$ & $\mathrm{~T}=2.00$ \\
& & & & & \\
\hline$(15,5)$ & 1 & $2.3844(0.87)$ & $2.3987(0.87)$ & $2.2207(0.91)$ & $2.1709(0.91)$ \\
& 2 & $3.6705(0.85)$ & $3.3235(0.83)$ & $2.8270(0.85)$ & $2.7371(0.88)$ \\
& 3 & $2.5863(0.87)$ & $2.5484(0.87)$ & $2.3481(0.92)$ & $2.2763(0.92)$ \\
\hline$(25,5)$ & 1 & $2.4053(0.84)$ & $2.2542(0.91)$ & $2.1722(0.91)$ & $2.1693(0.91)$ \\
& 2 & $4.0236(0.87)$ & $3.3161(0.84)$ & $2.7860(0.87)$ & $2.4084(0.90)$ \\
& 3 & $2.5294(0.87)$ & $2.3711(0.92)$ & $2.2754(0.92)$ & $2.2718(0.92)$ \\
\hline$(50,5)$ & 1 & $2.2460(0.91)$ & $2.1715(0.91)$ & $2.1693(0.91)$ & $2.1693(0.91)$ \\
& 2 & $3.9815(0.86)$ & $3.3038(0.85)$ & $2.7495(0.87)$ & $2.3903(0.89)$ \\
& 3 & $2.3522(0.92)$ & $2.2736(0.92)$ & $2.2718(0.92)$ & $2.2718(0.92)$ \\
\hline$(100,5)$ & 1 & $2.1712(0.91)$ & $2.1693(0.91)$ & $2.1693(0.91)$ & $2.1693(0.91)$ \\
& 2 & $3.9598(0.88)$ & $3.2870(0.85)$ & $2.7571(0.88)$ & $2.4100(0.89)$ \\
& 3 & $2.2728(0.92)$ & $2.2718(0.92)$ & $2.2718(0.92)$ & $2.2718(0.92)$ \\
\hline$(25,10)$ & 1 & $1.9711(0.85)$ & $1.5468(0.91)$ & $1.3986(0.93)$ & $1.3801(0.93)$ \\
& 2 & $2.9016(0.82)$ & $2.3629(0.88)$ & $1.8282(0.88)$ & $1.5380(0.91)$ \\
& 3 & $2.1145(0.85)$ & $1.6372(0.90)$ & $1.4341(0.91)$ & $1.3873(0.92)$ \\
\hline$(50,10)$ & 1 & $1.5219(0.90)$ & $1.3911(0.93)$ & $1.3800(0.93)$ & $1.3800(0.93)$ \\
& 2 & $2.9276(0.82)$ & $2.3171(0.87)$ & $1.8030(0.88)$ & $1.5254(0.91)$ \\
& 3 & $1.5494(0.91)$ & $1.4041(0.92)$ & $1.3854(0.92)$ & $1.3852(0.92)$ \\
\hline$(100,10)$ & 1 & $1.3889(0.93)$ & $1.3800(0.93)$ & $1.3800(0.93)$ & $1.3800(0.93)$ \\
& 2 & $2.8062(0.82)$ & $2.2391(0.86)$ & $1.7609(0.87)$ & $1.4959(0.91)$ \\
& 3 & $1.3949(0.92)$ & $1.3852(0.92)$ & $1.3852(0.92)$ & $1.3852(0.92)$ \\
\hline$(50,15)$ & 1 & $1.3523(0.88)$ & $1.1282(0.92)$ & $1.0910(0.93)$ & $1.0909(0.93)$ \\
& 2 & $2.4188(0.86)$ & $1.8103(0.87)$ & $1.4050(0.90)$ & $1.1958(0.92)$ \\
& 3 & $1.4193(0.88)$ & $1.1420(0.91)$ & $1.0876(0.93)$ & $1.0853(0.93)$ \\
\hline$(100,15)$ & 1 & $1.1213(0.93)$ & $1.0909(0.93)$ & $1.0909(0.93)$ & $1.0909(0.93)$ \\
& 2 & $2.4659(0.87)$ & $1.8500(0.89)$ & $1.4179(0.90)$ & $1.1981(0.93)$ \\
& 3 & $1.1209(0.92)$ & $1.0853(0.93)$ & $1.0853(0.93)$ & $1.0853(0.93)$ \\
\hline
\end{tabular}


Table 5: The average confidence lengths and the corresponding coverage percentages of the Bayes credible intervals for different sample sizes and for different schemes are presented. Each value represents the average confidence length and the corresponding coverage percentage is reported within bracket.

\begin{tabular}{|l|l|l|l|l|l|}
\hline$(n, m)$ & Scheme & $\mathrm{T}=0.25$ & $\mathrm{~T}=0.50$ & $\mathrm{~T}=1.00$ & $\mathrm{~T}=2.00$ \\
& & & & & \\
\hline$(15,5)$ & 1 & $2.3429(0.94)$ & $2.2510(0.94)$ & $2.2537(0.95)$ & $2.2538(0.95)$ \\
& 2 & $3.5213(0.96)$ & $2.8663(0.94)$ & $2.4443(0.93)$ & $2.2814(0.95)$ \\
& 3 & $2.4345(0.94)$ & $2.2641(0.93)$ & $2.2531(0.94)$ & $2.2538(0.95)$ \\
\hline$(25,5)$ & 1 & $2.2513(0.92)$ & $2.2527(0.95)$ & $2.2538(0.95)$ & $2.2538(0.95)$ \\
& 2 & $3.4957(0.96)$ & $2.8533(0.95)$ & $2.4365(0.93)$ & $2.2800(0.95)$ \\
& 3 & $2.2599(0.91)$ & $2.2529(0.94)$ & $2.2538(0.95)$ & $2.2538(0.95)$ \\
\hline$(50,5)$ & 1 & $2.2534(0.95)$ & $2.2538(0.95)$ & $2.2538(0.95)$ & $2.2538(0.95)$ \\
& 2 & $3.4661(0.96)$ & $2.8438(0.95)$ & $2.4360(0.93)$ & $2.2793(0.95)$ \\
& 3 & $2.2527(0.95)$ & $2.2538(0.95)$ & $2.2538(0.95)$ & $2.2538(0.95)$ \\
\hline$(100,5)$ & 1 & $2.2538(0.95)$ & $2.2538(0.95)$ & $2.2538(0.95)$ & $2.2538(0.95)$ \\
& 2 & $3.4549(0.96)$ & $2.8366(0.95)$ & $2.4349(0.93)$ & $2.2796(0.95)$ \\
& 3 & $2.2538(0.95)$ & $2.2538(0.95)$ & $2.2538(0.95)$ & $2.2538(0.95)$ \\
\hline$(25,10)$ & 1 & $1.6411(0.91)$ & $1.3802(0.95)$ & $1.3641(0.95)$ & $1.3641(0.95)$ \\
& 2 & $2.4135(0.95)$ & $1.9309(0.93)$ & $1.5949(0.94)$ & $1.4182(0.94)$ \\
& 3 & $1.7641(0.91)$ & $1.4503(0.94)$ & $1.3652(0.95)$ & $1.3641(0.95)$ \\
\hline$(50,10)$ & 1 & $1.3703(0.93)$ & $1.3641(0.95)$ & $1.3641(0.95)$ & $1.3641(0.95)$ \\
& 2 & $2.3853(0.95)$ & $1.9167(0.94)$ & $1.5883(0.94)$ & $1.4163(0.94)$ \\
& 3 & $1.3802(0.95)$ & $1.3641(0.95)$ & $1.3641(0.95)$ & $1.3641(0.95)$ \\
\hline$(100,10)$ & 1 & $1.3641(0.95)$ & $1.3641(0.95)$ & $1.3641(0.95)$ & $1.3641(0.95)$ \\
& 2 & $2.3709(0.95)$ & $1.9095(0.93)$ & $1.5866(0.94)$ & $1.4157(0.94)$ \\
& 3 & $1.3641(0.95)$ & $1.3641(0.95)$ & $1.3641(0.95)$ & $1.3641(0.95)$ \\
\hline$(50,15)$ & 1 & $1.1769(0.93)$ & $1.0722(0.95)$ & $1.0721(0.96)$ & $1.0721(0.96)$ \\
& 2 & $1.9624(0.92)$ & $1.5554(0.94)$ & $1.2748(0.94)$ & $1.1221(0.95)$ \\
& 3 & $1.2399(0.95)$ & $1.0776(0.95)$ & $1.0721(0.96)$ & $1.0721(0.96)$ \\
\hline$(100,15)$ & 1 & $1.0720(0.95)$ & $1.0721(0.96)$ & $1.0721(0.96)$ & $1.0721(0.96)$ \\
& 2 & $1.9454(0.93)$ & $1.5472(0.94)$ & $1.2717(0.94)$ & $1.1213(0.95)$ \\
& 3 & $1.0722(0.95)$ & $1.0721(0.96)$ & $1.0721(0.96)$ & $1.0721(0.96)$ \\
\hline
\end{tabular}


Table 6: The average confidence lengths and the corresponding coverage percentages of the confidence intervals based on the asymptotic distribution of $\ln \hat{\lambda}$ for different sample sizes and for different schemes are presented. Each value represents the average confidence length and the corresponding coverage percentage is reported within bracket.

\begin{tabular}{|l|l|l|l|l|l|}
\hline$(n, m)$ & Scheme & $\mathrm{T}=0.25$ & $\mathrm{~T}=0.50$ & $\mathrm{~T}=1.00$ & $\mathrm{~T}=2.00$ \\
& & & & & \\
\hline$(15,5)$ & 1 & $2.9168(0.91)$ & $2.6135(0.93)$ & $2.5973(0.93)$ & $2.5974(0.93)$ \\
& 2 & $5.3427(0.92)$ & $3.8316(0.94)$ & $2.9865(0.94)$ & $2.6637(0.93)$ \\
& 3 & $3.0859(0.91)$ & $2.6478(0.93)$ & $2.5977(0.93)$ & $2.5974(0.93)$ \\
\hline$(25,5)$ & 1 & $2.6297(0.93)$ & $2.5967(0.92)$ & $2.5974(0.93)$ & $2.5974(0.93)$ \\
& 2 & $5.2386(0.94)$ & $3.7872(0.94)$ & $2.9719(0.94)$ & $2.6612(0.93)$ \\
& 3 & $2.6521(0.93)$ & $2.5974(0.93)$ & $2.5974(0.93)$ & $2.5974(0.93)$ \\
\hline$(50,5)$ & 1 & $2.5971(0.93)$ & $2.5974(0.93)$ & $2.5974(0.93)$ & $2.5974(0.93)$ \\
& 2 & $5.1482(0.94)$ & $3.7573(0.94)$ & $2.9653(0.94)$ & $2.6597(0.93)$ \\
& 3 & $2.5967(0.92)$ & $2.5974(0.93)$ & $2.5974(0.93)$ & $2.5974(0.93)$ \\
\hline$(100,5)$ & 1 & $2.5974(0.93)$ & $2.5974(0.93)$ & $2.5974(0.93)$ & $2.5974(0.93)$ \\
& 2 & $5.1068(0.94)$ & $3.7413(0.94)$ & $2.9614(0.94)$ & $2.6595(0.93)$ \\
& 3 & $2.5974(0.93$ & $2.5974(0.93$ & $2.5974(0.93$ & $2.5974(0.93$ \\
\hline$(25,10)$ & 1 & $1.8833(0.97)$ & $1.4940(0.95)$ & $1.4658(0.95)$ & $1.4658(0.95)$ \\
& 2 & $3.1850(0.95)$ & $2.2836(0.96)$ & $1.7772(0.96)$ & $1.5378(0.95)$ \\
& 3 & $2.0505(0.96)$ & $1.5880(0.95)$ & $1.4683(0.95)$ & $1.4658(0.95)$ \\
\hline$(50,10)$ & 1 & $1.4789(0.95)$ & $1.4658(0.95)$ & $1.4658(0.95)$ & $1.4658(0.95)$ \\
& 2 & $3.1027(0.95)$ & $2.2566(0.95)$ & $1.7682(0.95)$ & $1.5355(0.95)$ \\
& 3 & $1.4940(0.95)$ & $1.4658(0.95)$ & $1.4658(0.95)$ & $1.4658(0.95)$ \\
\hline$(100,10)$ & 1 & $1.4658(0.95)$ & $1.4658(0.95)$ & $1.4658(0.95)$ & $1.4658(0.95)$ \\
& 2 & $3.0659(0.95)$ & $2.2439(0.95)$ & $1.7652(0.95)$ & $1.5347(0.95)$ \\
& 3 & $1.4658(0.95)$ & $1.4658(0.95)$ & $1.4658(0.95)$ & $1.4658(0.95)$ \\
\hline$(50,15)$ & 1 & $1.2593(0.95)$ & $1.1254(0.95)$ & $1.1250(0.96)$ & $1.1250(0.96)$ \\
& 2 & $2.3907(0.97)$ & $1.7463(0.95)$ & $1.3721(0.95)$ & $1.1852(0.95)$ \\
& 3 & $1.3364(0.96)$ & $1.1325(0.95)$ & $1.1250(0.96)$ & $1.1250(0.96)$ \\
\hline$(100,15)$ & 1 & $1.1250(0.96)$ & $1.1250(0.96)$ & $1.1250(0.96)$ & $1.1250(0.96)$ \\
& 2 & $2.3570(0.97)$ & $1.7346(0.95)$ & $1.3683(0.95)$ & $1.1843(0.95)$ \\
& 3 & $1.1253(0.95)$ & $1.1250(0.95)$ & $1.1250(0.95)$ & $1.1250(0.95)$ \\
\hline
\end{tabular}


Table 7: The average confidence lengths and the corresponding coverage percentages of the confidence intervals based on the likelihood ratio test, for different sample sizes and for different schemes are presented. Each value represents the average confidence length and the corresponding coverage percentage is reported within bracket.

\begin{tabular}{|l|l|l|l|l|l|}
\hline$(n, m)$ & Scheme & $\mathrm{T}=0.25$ & $\mathrm{~T}=0.50$ & $\mathrm{~T}=1.00$ & $\mathrm{~T}=2.00$ \\
& & & & & \\
\hline$(15,5)$ & 1 & $2.5319(0.96)$ & $2.3436(0.93)$ & $2.3419(0.94)$ & $2.3420(0.94)$ \\
& 2 & $4.0022(0.97)$ & $3.0944(0.95)$ & $2.5791(0.93)$ & $2.3789(0.94)$ \\
& 3 & $2.6445(0.96)$ & $2.3617(0.93)$ & $2.3415(0.94)$ & $2.3420(0.94)$ \\
\hline$(25,5)$ & 1 & $2.3475(0.94)$ & $2.3410(0.94)$ & $2.3420(0.94)$ & $2.3420(0.94)$ \\
& 2 & $3.8857(0.96)$ & $3.0749(0.95)$ & $2.5697(0.93)$ & $2.3772(0.94)$ \\
& 3 & $2.3592(0.94)$ & $2.3413(0.94)$ & $2.3420(0.94)$ & $2.3420(0.94)$ \\
\hline$(50,5)$ & 1 & $2.3416(0.94)$ & $2.3420(0.94)$ & $2.3420(0.94)$ & $2.3420(0.94)$ \\
& 2 & $3.8440(0.97)$ & $3.0610(0.95)$ & $2.5680(0.94)$ & $2.3764(0.94)$ \\
& 3 & $2.3410(0.94)$ & $2.3420(0.94)$ & $2.3420(0.94)$ & $2.3420(0.94)$ \\
\hline$(100,5)$ & 1 & $2.3420(0.94)$ & $2.3420(0.94)$ & $2.3420(0.94)$ & $2.3420(0.94)$ \\
& 2 & $3.8268(0.97)$ & $3.0519(0.95)$ & $2.5662(0.94)$ & $2.3765(0.94)$ \\
& 3 & $2.3420(0.94)$ & $2.3420(0.94)$ & $2.3420(0.94)$ & $2.3420(0.94)$ \\
\hline$(25,10)$ & 1 & $1.7026(0.95)$ & $1.4101(0.94)$ & $1.3912(0.94)$ & $1.3912(0.94)$ \\
& 2 & $2.5959(0.96)$ & $2.0194(0.94)$ & $1.6422(0.94)$ & $1.4496(0.95)$ \\
& 3 & $1.8365(0.95)$ & $1.4862(0.94)$ & $1.3927(0.95)$ & $1.3912(0.94)$ \\
\hline$(50,10)$ & 1 & $1.3990(0.95)$ & $1.3912(0.94)$ & $1.3912(0.94)$ & $1.3912(0.94)$ \\
& 2 & $2.5564(0.96)$ & $2.0023(0.94)$ & $1.6349(0.94)$ & $1.4477(0.94)$ \\
& 3 & $1.4102(0.94)$ & $1.3912(0.94)$ & $1.3912(0.94)$ & $1.3912(0.94)$ \\
\hline$(100,10)$ & 1 & $1.3912(0.94)$ & $1.3912(0.94)$ & $1.3912(0.94)$ & $1.3912(0.94)$ \\
& 2 & $2.5372(0.95)$ & $1.9937(0.94)$ & $1.6330(0.94)$ & $1.4470(0.94)$ \\
& 3 & $1.3912(0.94)$ & $1.3912(0.94)$ & $1.3912(0.94)$ & $1.3912(0.94)$ \\
\hline$(50,15)$ & 1 & $1.1987(0.95)$ & $1.0864(0.95)$ & $1.0862(0.95)$ & $1.0862(0.95)$ \\
& 2 & $2.0680(0.95)$ & $1.6046(0.95)$ & $1.3005(0.95)$ & $1.1389(0.95)$ \\
& 3 & $1.2654(0.95)$ & $1.0922(0.95)$ & $1.0862(0.96)$ & $1.0862(0.96)$ \\
\hline$(100,15)$ & 1 & $1.0861(0.95)$ & $1.0862(0.95)$ & $1.0862(0.95)$ & $1.0862(0.95)$ \\
& 2 & $2.0472(0.95)$ & $1.5956(0.95)$ & $1.2972(0.95)$ & $1.1381(0.95)$ \\
& 3 & $1.0864(0.95)$ & $1.0862(0.95)$ & $1.0862(0.95)$ & $1.0862(0.95)$ \\
\hline
\end{tabular}


Table 8: Total probabilities $\left[\sum_{i=1}^{m} P\left(X_{i: m: n}<T\right)\right]$ are reported for each scheme and for each case. Moreover $\mathrm{P}$ (Case I terminated) and the expected duration of the experiments are reported within brackets as the first figure and the second figure respectively.

Table 8a: Here $n=15, m=5$.

\begin{tabular}{|r|r|r|r|r|}
\hline Scheme & $\mathrm{T}=0.25$ & $\mathrm{~T}=0.50$ & $\mathrm{~T}=1.00$ & $\mathrm{~T}=2.00$ \\
& & & & \\
\hline Scheme-1 & $3.27(0.25,0.23)$ & $4.66(0.78,0.36)$ & $5.00(1.00,0.39)$ & $5.00(1.00,0.39)$ \\
Scheme-2 & $1.67(0.00,0.25)$ & $2.42(0.02,0.50)$ & $3.45(0.14,0.96)$ & $4.45(0.55,1.61)$ \\
Scheme-3 & $2.99(0.14,0.24)$ & $4.37(0.57,0.41)$ & $4.96(0.96,0.48)$ & $5.00(1.00,0.48)$ \\
Scheme-4 & $2.68(0.04,0.25)$ & $3.90(0.28,0.46)$ & $4.74(0.77,0.68)$ & $4.98(0.98,0.75)$ \\
\hline
\end{tabular}

Table 8b: Here $n=25, m=5$.

\begin{tabular}{|r|r|r|r|r|}
\hline Scheme & $\mathrm{T}=0.25$ & $\mathrm{~T}=0.50$ & $\mathrm{~T}=1.00$ & $\mathrm{~T}=2.00$ \\
\hline Scheme-1 & $4.48(0.70,0.19)$ & $4.99(0.99,0.22)$ & $5.00(1.00,0.22)$ & $5.00(1.00,0.22)$ \\
Scheme-2 & $1.78(0.00,0.25)$ & $2.50(0.02,0.50)$ & $3.50(0.15,0.96)$ & $4.46(0.56,1.60)$ \\
Scheme-3 & $4.34(0.62,0.20)$ & $4.97(0.98,0.24)$ & $5.00(1.00,0.24)$ & $5.00(1.00,0.24)$ \\
Scheme-4 & $4.14(0.50,0.21)$ & $4.93(0.95,0.27)$ & $5.00(1.00,0.28)$ & $5.00(1.00,0.28)$ \\
Scheme-5 & $3.92(0.38,0.22)$ & $4.84(0.87,0.31)$ & $5.00(1.00,0.34)$ & $5.00(1.00,0.33)$ \\
Scheme-6 & $3.61(0.21,0.24)$ & $4.61(0.67,0.38)$ & $4.96(0.96,0.45)$ & $5.00(1.00,0.45)$ \\
Scheme-7 & $3.27(0.03,0.25)$ & $4.04(0.18,0.47)$ & $4.47(0.48,0.81)$ & $4.80(0.80,1.11)$ \\
\hline
\end{tabular}

Table 8c: Here $n=50, m=5$.

\begin{tabular}{|r|r|r|r|r|}
\hline Scheme & $\mathrm{T}=0.25$ & $\mathrm{~T}=0.50$ & $\mathrm{~T}=1.00$ & $\mathrm{~T}=2.00$ \\
\hline Scheme-1 & $4.99(0.99,0.10)$ & $5.00(1.00,0.10)$ & $5.00(1.00,0.10)$ & $5.00(1.00,0.10)$ \\
Scheme-2 & $1.83(0.00,0.25)$ & $2.56(0.02,0.50)$ & $3.53(0.16,0.95)$ & $4.47(0.56,1.59)$ \\
Scheme-3 & $4.98(0.99,0.11)$ & $5.00(1.00,0.11)$ & $5.00(1.00,0.11)$ & $5.00(1.00,0.11)$ \\
Scheme-4 & $4.97(0.98,0.11)$ & $5.00(1.00,0.11)$ & $5.00(1.00,0.11)$ & $5.00(1.00,0.11)$ \\
Scheme-5 & $4.97(0.98,0.12)$ & $5.00(1.00,0.12)$ & $5.00(1.00,0.12)$ & $5.00(1.00,0.12)$ \\
Scheme-6 & $4.95(0.96,0.12)$ & $5.00(1.00,0.13)$ & $5.00(1.00,0.13)$ & $5.00(1.00,0.13)$ \\
Scheme-7 & $4.93(0.94,0.12)$ & $5.00(1.00,0.14)$ & $5.00(1.00,0.14)$ & $5.00(1.00,0.14)$ \\
Scheme-8 & $4.90(0.91,0.14)$ & $5.00(1.00,0.15)$ & $5.00(1.00,0.15)$ & $5.00(1.00,0.15)$ \\
\hline
\end{tabular}


Table 8d: Here $n=100, m=5$.

\begin{tabular}{|r|r|r|r|r|}
\hline Scheme & $\mathrm{T}=0.25$ & $\mathrm{~T}=0.50$ & $\mathrm{~T}=1.00$ & $\mathrm{~T}=2.00$ \\
& & & & \\
\hline Scheme-1 & $5.00(1.00,0.05)$ & $5.00(1.00,0.05)$ & $5.00(1.00,0.05)$ & $5.00(1.00,0.05)$ \\
Scheme-2 & $1.87(0.00,0.25)$ & $2.56(0.02,0.50)$ & $3.54(0.16,0.95)$ & $4.47(0.56,1.58)$ \\
Scheme-3 & $5.00(1.00,0.05)$ & $5.00(1.00,0.05)$ & $5.00(1.00,0.05)$ & $5.00(1.00,0.05)$ \\
Scheme-4 & $5.00(1.00,0.05)$ & $5.00(1.00,0.05)$ & $5.00(1.00,0.05)$ & $5.00(1.00,0.05)$ \\
Scheme-5 & $5.00(1.00,0.05)$ & $5.00(1.00,0.05)$ & $5.00(1.00,0.05)$ & $5.00(1.00,0.05)$ \\
Scheme-6 & $5.00(1.00,0.05)$ & $5.00(1.00,0.05)$ & $5.00(1.00,0.05)$ & $5.00(1.00,0.05)$ \\
Scheme-7 & $5.00(1.00,0.06)$ & $5.00(1.00,0.06)$ & $5.00(1.00,0.06)$ & $5.00(1.00,0.06)$ \\
Scheme-8 & $5.00(1.00,0.06)$ & $5.00(1.00,0.06)$ & $5.00(1.00,0.06)$ & $5.00(1.00,0.06)$ \\
\hline
\end{tabular}

Table 8e: Here $n=25, m=10$.

\begin{tabular}{|r|r|r|r|r|}
\hline Scheme & $\mathrm{T}=0.25$ & $\mathrm{~T}=0.50$ & $\mathrm{~T}=1.00$ & $\mathrm{~T}=2.00$ \\
& & & & \\
\hline Scheme-1 & $5.50(0.03,0.25)$ & $8.89(0.52,0.44)$ & $9.99(0.99,0.51)$ & $10.00(1.00,0.49)$ \\
Scheme-2 & $2.70(0.00,0.25)$ & $4.29(0.00,0.50)$ & $6.48(0.01,1.00)$ & $8.75(0.25,1.89)$ \\
Scheme-3 & $4.92(0.01,0.25)$ & $7.76(0.18,0.49)$ & $9.76(0.82,0.72)$ & $10.00(1.00,0.73)$ \\
\hline
\end{tabular}

Table 8f: Here $n=50, m=10$.

\begin{tabular}{|r|r|r|r|r|}
\hline Scheme & $\mathrm{T}=0.25$ & $\mathrm{~T}=0.50$ & $\mathrm{~T}=1.00$ & $\mathrm{~T}=2.00$ \\
& & & & \\
\hline Scheme-1 & $9.35(0.70,0.20)$ & $10.00(1.00,0.22)$ & $10.00(1.00,0.22)$ & $10.00(1.00,0.22)$ \\
Scheme-2 & $2.86(0.00,0.25)$ & $4.41(0.00,0.50)$ & $6.57(0.01,1.00)$ & $8.77(0.27,1.88)$ \\
Scheme-3 & $9.00(0.55,0.22)$ & $9.99(0.99,0.25)$ & $10.00(1.00,0.25)$ & $10.00(1.00,0.25)$ \\
Scheme-4 & $8.47(0.36,0.23)$ & $9.97(0.97,0.29)$ & $10.00(1.00,0.29)$ & $10.00(1.00,0.29)$ \\
Scheme-5 & $7.84(0.16,0.24)$ & $9.79(0.84,0.36)$ & $10.00(1.00,0.37)$ & $10.00(1.00,0.37)$ \\
Scheme-6 & $7.11(0.03,0.25)$ & $9.18(0.41,0.45)$ & $9.93(0.93,0.58)$ & $10.00(1.00,0.58)$ \\
\hline
\end{tabular}

Table 8g: Here $n=100, m=10$.

\begin{tabular}{|r|r|r|r|r|}
\hline Scheme & $\mathrm{T}=0.25$ & $\mathrm{~T}=0.50$ & $\mathrm{~T}=1.00$ & $\mathrm{~T}=2.00$ \\
& & & & \\
\hline Scheme-1 & $10.00(1.00,0.10)$ & $10.00(1.00,0.10)$ & $10.00(1.00,0.10)$ & $10.00(1.00,0.10)$ \\
Scheme-2 & $2.93(0.00,0.25)$ & $4.48(0.00,0.50)$ & $6.59(0.01,1.00)$ & $8.79(0.27,1.88)$ \\
Scheme-3 & $10.00(1.00,0.11)$ & $10.00(1.00,0.11)$ & $10.00(1.00,0.11)$ & $10.00(1.00,0.11)$ \\
Scheme-4 & $10.00(1.00,0.12)$ & $10.00(1.00,0.12)$ & $10.00(1.00,0.12)$ & $10.00(1.00,0.12)$ \\
Scheme-5 & $10.00(1.00,0.12)$ & $10.00(1.00,0.12)$ & $10.00(1.00,0.12)$ & $10.00(1.00,0.12)$ \\
Scheme-6 & $9.98(0.99,0.13)$ & $10.00(1.00,0.13)$ & $10.00(1.00,0.13)$ & $10.00(1.00,0.13)$ \\
Scheme-7 & $9.96(0.97,0.15)$ & $10.00(1.00,0.15)$ & $10.00(1.00,0.15)$ & $10.00(1.00,0.15)$ \\
Scheme-8 & $9.93(0.94,0.16)$ & $10.00(1.00,0.16)$ & $10.00(1.00,0.16)$ & $10.00(1.00,0.16)$ \\
\hline
\end{tabular}


Table 8h: Here $n=50, m=15$.

\begin{tabular}{|r|r|r|r|r|}
\hline Scheme & $\mathrm{T}=0.25$ & $\mathrm{~T}=0.50$ & $\mathrm{~T}=1.00$ & $\mathrm{~T}=2.00$ \\
& & & & \\
\hline Scheme-1 & $10.88(0.12,0.25)$ & $14.87(0.94,0.35)$ & $15.00(1.00,0.35)$ & $15.00(1.00,0.35)$ \\
Scheme-2 & $3.82(0.00,0.25)$ & $6.32(0.00,0.50)$ & $9.75(0.00,1.00)$ & $13.09(0.12,1.96)$ \\
Scheme-3 & $9.75(0.02,0.25)$ & $14.40(0.69,0.42)$ & $15.00(1.00,0.44)$ & $15.00(1.00,0.44)$ \\
Scheme-4 & $8.72(0.00,0.25)$ & $12.89(0.18,0.49)$ & $14.91(0.92,0.67)$ & $15.00(1.00,0.68)$ \\
\hline
\end{tabular}

Table 8i: Here $n=100, m=15$.

\begin{tabular}{|r|r|r|r|r|}
\hline Scheme & $\mathrm{T}=0.25$ & $\mathrm{~T}=0.50$ & $\mathrm{~T}=1.00$ & $\mathrm{~T}=2.00$ \\
& & & & \\
\hline Scheme-1 & $14.95(0.98,0.16)$ & $15.00(1.00,0.16)$ & $15.00(1.00,0.16)$ & $15.00(1.00,0.16)$ \\
Scheme-2 & $3.93(0.00,0.25)$ & $6.42(0.00,0.50)$ & $9.80(0.00,1.00)$ & $13.11(0.12,1.96)$ \\
Scheme-3 & $14.87(0.94,0.18)$ & $15.00(1.00,0.18)$ & $15.00(1.00,0.18)$ & $15.00(1.00,0.18)$ \\
Scheme-4 & $14.72(0.86,0.19)$ & $15.00(1.00,0.19)$ & $15.00(1.00,0.19)$ & $15.00(1.00,0.19)$ \\
Scheme-5 & $14.43(0.71,0.21)$ & $15.00(1.00,0.22)$ & $15.00(1.00,0.22)$ & $15.00(1.00,0.22)$ \\
Scheme-6 & $13.80(0.46,0.22)$ & $14.99(0.99,0.26)$ & $15.00(1.00,0.26)$ & $15.00(1.00,0.26)$ \\
Scheme-7 & $12.87(0.18,0.24)$ & $14.88(0.90,0.34)$ & $15.00(1.00,0.34)$ & $15.00(1.00,0.34)$ \\
Scheme-8 & $11.78(0.01,0.25)$ & $13.90(0.19,0.48)$ & $14.67(0.67,0.75)$ & $14.97(0.97,0.87)$ \\
\hline
\end{tabular}

